\title{
Forensics Analysis from Cloud Storage Client Application on Proprietary Operating System
}

\author{
Amirullah Amirullah \\ Universitas Islam Indonesia \\ Jl. Kaliurang KM 14,5 \\ Yogyakarta, 55584
}

\author{
Imam Riadi \\ Universitas Ahmad Dahlan \\ Jl. Prof. Dr. Soepomo, \\ Yogyakarta, 55164
}

\author{
Ahmad Luthfi \\ Universitas Islam Indonesia \\ Jl. Kaliurang KM 14,5 \\ Yogyakarta, 55584
}

\begin{abstract}
Increased use of cloud storage services have become a necessity alternative that complements the main storage media in everyday activities because it offers ease of doing automatic backups, sharing files and photos and so forth on a variety of computing devices and smartphones. It is very possible loopholes for criminals to store illegal files or matters relating to such activities. There are various types of cloud services with each type having a different potential use in criminal activities. One area of difficulty is the identification and acquisition of potential data when different services can be exploited by criminals. Because geographically cloud servers scattered in various regions. This causes difficulties for digital forensic investigators will add time and expense, to contact all potential service providers to determine whether the data stored in the server cloud services. This paper presented at a target on the client side user application to help find the data renmant on the use of cloud storage applications of various service provider on the proprietary operating system focuses on the Windows 10 platform. Results from this research include a variety of state after the install, deleted and uninstall web browser and memory, in order to find digital evidence. Based on the test results the success index average was $82.63 \%$ and the remaining can not be analyzed, the results depend on various state, procedures and tools that are used, the research can be carried out smoothly.
\end{abstract}

\section{General Terms}

Digital investigation, Cloud storage, Application Forensics.

\section{Keywords}

Cloud, Storage, Forensics, Operating System, Windows 10.

\section{INTRODUCTION}

Fifth Annual Report of the Cisco (B) Global Cloud Index (2014-2019), which was released October 28, 2015 forecasts that global cloud traffic will more than quadruple by the end of 2019 , from 2.1 to 8.6 zettabytes (ZB), outpacing the growth of total global data center traffic, which is forecast to triple during the same time frame (from 3.4 to $10.4 \mathrm{ZB}$ ). Several factors are driving cloud traffic's accelerating growth and the transition to cloud services, including the personal cloud demands of an increasing number of mobile devices; the rapid growth in popularity of public cloud services for business, and the increased degree of virtualization in private clouds which is increasing the density of those workloads. The growth of machine-to-machine (M2M) connections also has the potential to drive more cloud traffic in the future [1].

Cisco GCI (Global Cloud Index) estimates that in 2019, more than half or 55 percent ( 2 billion) of the population of Internet consumers will use personal cloud storage, up from 42 percent (1.1 billion users) by 2014. as shown in the Fig 1 .

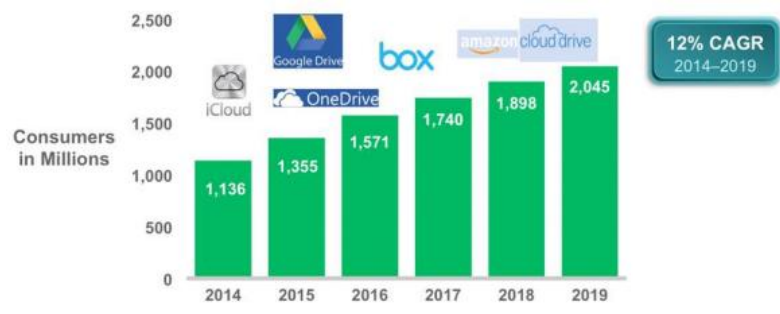

Fig 1: Expansion Cloud Storage Consumer

Cisco GCI forecasts that global consumer cloud storage traffic will grow from 14 EB annually in 2014 to 39 EB by 2019 at a Compound Annual Grade Rate (CAGR) of 23 percent (Fig. 2). This growth translates to per-user traffic of 1.6 Gigabytes (GB) per month by 2019, compared to $992 \mathrm{MB}$ per month in 2014 [2].

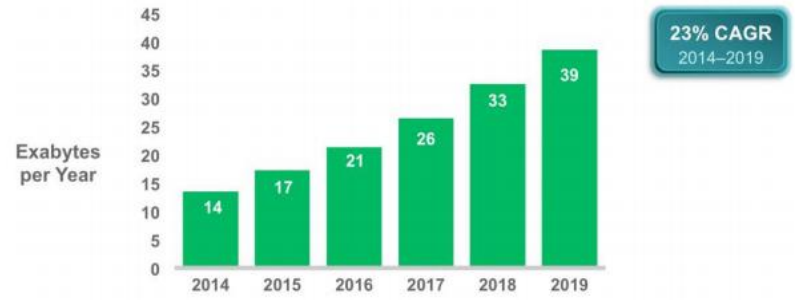

Fig 2: Consumer Cloud Storage Traffic

There are two things associated with cloud storage account. The first is related to the use of a web browser and the second is through a client application installed by the user's device. This research will conduct an analysis of several client applications and develop how to get the data. Previous research analysis applications on onedrive, google drive, and dropbox on windows 7 [3][4][5] and in Windows 8 [6][7], the research will be shown some of the client application that runs on Windows 10. to add differences and develop a different sample, the selected application is different from previous research. Then choose 3 cloud storage applications that Dropbox, Copy, and CloudMe. Specification of the three shown in Table 1 . 
Tab1e 1. Comparation DropBox, Copy and CloudMe

\begin{tabular}{|c|c|c|c|}
\hline \multicolumn{4}{|l|}{ Specifications } \\
\hline Version & 2.5 .35 & 1.44 & 1.9 .1 \\
\hline Operating Systems & WindowMacLinux & WindowM acLinux & WindowMacLinux \\
\hline Storage & $1 \mathrm{~TB}$ & $250 \mathrm{~GB}$ & $100 \mathrm{~GB}$ \\
\hline Monthly Price & $\$ 9.99$ & $\$ 4.99$ & $\$ 9$ \\
\hline Upload (Backup) Speed & Max (7.6 Mbps) & $7.8 \mathrm{Mbps}$ & $1.8 \mathrm{Mbps}$ \\
\hline Download (Restore) Speed & Max (30 Mbps) & $27 \mathrm{Mbps}$ & $74.7 \mathrm{Mbps}$ \\
\hline \multicolumn{4}{|l|}{ Features } \\
\hline Automatic Backup & yes & yes & yes \\
\hline Backup Any Folder & no & yes & yes \\
\hline Backup to Local Drive & no & no & no \\
\hline Free Trial & yes & yes & yes \\
\hline Free Online Storage & $2 \mathrm{~GB}$ & $15 \mathrm{~GB}$ & $3 \mathrm{~GB}$ \\
\hline File Versioning & yes & yes & no \\
\hline Keep Deleted Files & 30 Days & 30 Days & 60 Days \\
\hline Bandwidth Controls & yes & yes & yes \\
\hline Public File Sharing & yes & yes & yes \\
\hline NAS Backup & no & no & yes \\
\hline \multicolumn{4}{|l|}{ Security } \\
\hline Encrypted Storage & yes & yes & no \\
\hline Encrypted Transfer & yes & yes & yes \\
\hline Personal Encryption & no & no & no \\
\hline \multicolumn{4}{|l|}{ Support } \\
\hline Phone Support & yes & no & no \\
\hline Email Support & yes & yes & yes \\
\hline $24 / 7$ Support & no & no & no \\
\hline Live Chat & no & no & no \\
\hline
\end{tabular}

Previously study about the features and security in the CloudMe, and Dropbox on research [8], examined the security mechanisms of cloud storage services identified four typical features of cloud storage services, the copy, backup, synchronization and file sharing feature. Examined [9] various cloud storage vendors provides data storage, space availability, scaling, sharing, secure transmission between. cloud storage medium. In the above table is a comparison of specifications, features, security and support [10], major difference with the other in file versioning, keep deleted files, and encrypted storage. it is to understand the depth of the object of study that will be discussed, and this is part of the preparation phase.

\section{RELATED WORK}

Reese [11]describes the process using a snapshot using Boot EBS volume within Amazon's cloud services where there is the ability to snapshot a system, however, this does not apply to service Cloud storage as this process is beyond the control of the user. Iswardani. A, [12] examined log for denial of service attack in cloud. Clark, [13] examined the image Exif metadata remnants on Microsoft SkyDrive, Windows Azure, and Flickr, with a focus on information in the picture to be openly shared, found that personal information is available in a lot of pictures along with the public, which could be relevant in investigations, such as global positioning information. D. Quick, B. Martini, and K.-K. R. Choo [3] Examining the various circumstances that included a variety of methods to store, upload, and access data in the cloud. Potential sources of information are identified including the client $\log$ file software, prefetch files, link files, network traffic and memory capture, On Dropbox, Microsoft SkyDrive and Google Drive on Windows 7 and Apple iPhone 3G. AK McCurdy, Researching monitoring system that can be deployed and used by system administrators to detect the use Dropbox on the network, and broswer At the Dropbox application and OS Windows XP. [14]. S. Mehreen and B. Aslam [6] Examine artifacts dropbox on Windows 8, using digital forensic methodology.M. Epiphany [4] examined the artifacts Cloud Cloud storage on Dropbox, SkyDrive, Google Drive, iCloud operating system Windows 7. Chung [15] developed a procedure for the investigation of devices such as PCs and smartphones on Amazon S3, Dropbox, Google Docs and Evernote on Windows 7. Malik [7] Windows 8.1 researching digital artifacts in ownCloud, Copy, and Dropbox.

\section{METHODOLOGY}

The methodology used is the digital forensics methodology, namely preparation, preservation, analysis and reporting. The analysis method to find out all the problems and needs required to undertake to do the research thesis titled cloud storage application forensic methods to be used to look for problems, responding to the problems encountered and the testing stage as well as all the necessities such as needs analysis software and analysis of hardware requirements. Additionally two methods to answer research questions first used, among others: Methods Dynamic on Storage used for processes that occur during the installation and uninstall, activity of hard disks by using the tools Diskpulse, Regshot, Procces Monitor, and Procces Explorer, thus easily find artifacts location of the file or directory and characteristics or behavior of the application client Cloud Storage and Static methods used in the analysis of web browser and client database applications by conducting inspections analysis directly on the database files using sqlite browser, log viewer, do recovey deleted files can thus find artifacts related to client Cloud Storage. Coverage area of research is shown in Fig 3 below.

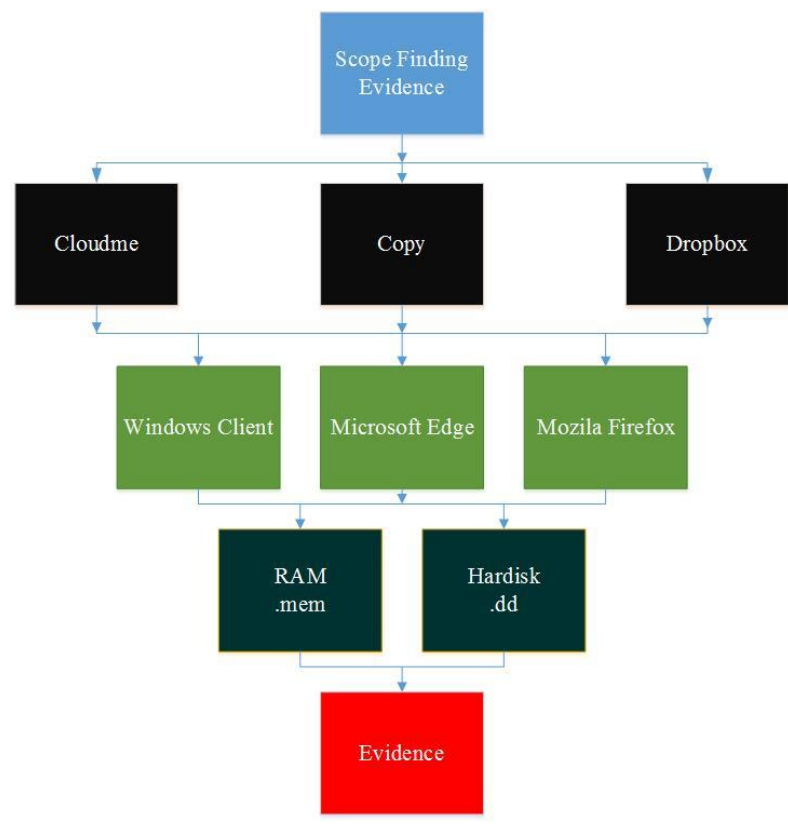

Fig 3: Block diagram of Research

In the picture above is a diagram of the research consists of three objects research is CloudMe, Copy, and Dropbox third Cloud Storage is accessed through the Windows client, Microsoft Edge, and Mozilla Firefox then performed phase of preservation to obtain an image of the hard disk and RAM then taken to the next process for phase of analysis.

\section{TOOLS AND SETUP}

Software is the object of the research are shown in Table 2 below [16][17][18]. This is research limitation, any prior 
version of software may have difference outcomes, and subsequent changes to software my result in difference findings.

Table 2. Software Test

\begin{tabular}{|l|l|}
\hline \multicolumn{1}{|c|}{ Software } & \multicolumn{1}{c|}{ Version } \\
\hline Windows OS & 10 Pro \\
\hline Microsoft Edge & 20.10240 .16384 .0 \\
\hline Mozila Firefox & 41.0 .2 \\
\hline Dropbox Client & 3.10 .7 \\
\hline Copy Client & 3.2 .01 .0481 \\
\hline CloudMe Client & 1.9 .1 \\
\hline
\end{tabular}

The sample scenario deleted files uploaded and shown in Table 3.

Table 3. Sample File

\begin{tabular}{|l|l|l|}
\hline File Name & File Size (Bytes) & Deleted \\
\hline 1.jpg & 879394 & $1 \mathrm{MF}$ \\
\hline 2.jpg & 845941 & $2 \mathrm{MF}$ \\
\hline 3.mp4 & 39624188 & $3 \mathrm{MF}$ \\
\hline 4.jpg & 595284 & $1 \mathrm{ME}$ \\
\hline 5.jpg & 707105 & $2 \mathrm{ME}$ \\
\hline 6.xlsx & 118272 & $3 \mathrm{ME}$ \\
\hline 7.docx & 491330 & $1 \mathrm{CP}$ \\
\hline 8.mp3 & 5411654 & $2 \mathrm{CM}$ \\
\hline 9.xlx & 198144 & $3 \mathrm{DB}$ \\
\hline 10.pdf & 8124477 & - \\
\hline 11.jpg & 48852 & - \\
\hline
\end{tabular}

\section{Notes:}

$1 *=$ Copy $2 *=$ CloudMe $3 *=$ Drobpox $*$ MF=Mozila Firefox *ME$=$ Microsoft Edge $* \mathrm{CM}=\mathrm{Client}$ CloudMe $* \mathrm{CP}=\mathrm{Client}$ Copy $* \mathrm{DB}=$ Client Dropbox

There are eleven uploaded file on each server cloud, then given the elimination of the different circumstances in each file in a web browser and client-side applications, it's aiming to distinguish whether the synchronization process is going well. Also to simplify the process of finding evidence of the result of the elimination of those files.

\section{RESULT}

\subsection{Web Browser Analysis}

\subsubsection{Mozilla Firefox}

Web browser analysis is a process Analysis of the use of a web browser then that becomes the source of the evidence can be found in several databases. Mozilla Firefox can be found on a file folder \img_cloudtest1.dd\Users\cloudtest2015\AppData|Roaming \ MozillalFirefox\Profiles\p662qtt.default.

In Table 4 is shown the names of the database on Mozilla Firefox and its Description.

Table 4. File database Mozilla Firefox

\begin{tabular}{|l|l|}
\hline \multicolumn{1}{|c|}{ Name Database } & \multicolumn{1}{c|}{ Description } \\
\hline content-prefs.sqlite & Individual settings for pages. \\
\hline cookies.sqlite & Cookies \\
\hline formhistory.sqlite & Saved form data \\
\hline healthreport.sqlite & $\begin{array}{l}\text { For all bugs involving collection, } \\
\text { submission, analysis and user- } \\
\text { facing features } \\
\text { (about:healthreport) as part of the } \\
\text { Firefox Health Report product }\end{array}$ \\
\hline permissions.sqlite & $\begin{array}{l}\text { Permission database for cookies, } \\
\text { pop-up blocking, image loading } \\
\text { and add-ons installation. }\end{array}$ \\
\hline places.sqlite & $\begin{array}{l}\text { Bookmarks, browsing and } \\
\text { download history }\end{array}$ \\
\hline
\end{tabular}

Browser use Mozilla Firefox on cookiess.sqlite database stores information such as the base domain visited cloudme.com, copy.com and dropbox.com, saving time start of a connection, hostname, time expired, the time and the time the connection is terminated. Formhistory database stores information form field such as access login password, name, address and others. Places.sqlite database stores information about bookmarks, history of visits, information download the file name, storage location, connection time, hostname and favicons. File key3.db store information related to the encryption password database to decrypt the password can use firepasswordviewer from securityexploded.com

\subsubsection{Microsoft Edge}

Analysis of the use of a web browser then that becomes the source of the evidence can be found in a file databases. At Microsoft Edge can be found on a file limg_cloudtest1.dd\Userslcloudtest2015\AppDatalLocal\Micr osoft \Windows $\mid$ webcachelWebCacheV01.dat

In Table 5 is shown the names of the database on Mozilla Firefox and its Description.

Table 5. Table File ESE Microsoft Edge

\begin{tabular}{|l|l|}
\hline \multicolumn{1}{|c|}{ Name Table } & \multicolumn{1}{c|}{ Description } \\
\hline Content & Temporary internet Files \\
\hline Cookies & Cookies \\
\hline Doomstore & WebStore \\
\hline History & URL History \\
\hline Iedownload & Downloaded files \\
\hline MSHist01YYYYMMDD & Time span of URL History \\
\hline
\end{tabular}


In Table 5, Table Content contains information about temporary internet file consists of several containerid contained how much data is stored is stored, containerid could not a fixed value but can be capricious or random, tables cookies store information cookies stored on some containers, as well as the history table containing information browsing history and ieDownload information about the location of the downloaded file, the possibility of users use the browser to download files from the Microsoft edge CloudMe, Copy or Dropbox account. The password can descrypt using iepassview from nirsoft.com.

\subsection{Client Application Analysis}

\subsubsection{CloudMe}

CloudMe client during the installation and running of applications using the Disk pulse and Prosess Monitor easily find where the main file CloudMe stored during the analysis found important files related or could be evidence.

The main database file contained in the CloudMe /img_cloudtest1.dd/Users/cloudtest2015/AppData/Local/Clou dMe/Sync/cache.db

Fig 4 is shown the location on the path to the directory where the $\log$ files and database CloudMe.

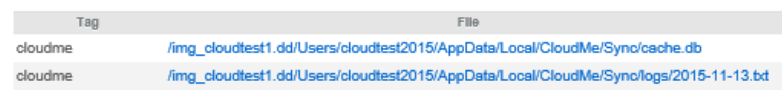

Fig 4: Location File of CloudMe

In Table 6 is shown the names of the database and log on CloudMe and its Description.

Table 6. File Analysis of CloudMe

\begin{tabular}{|l|l|}
\hline \multicolumn{1}{|c|}{ Name File } & \multicolumn{1}{|c|}{ Description } \\
\hline \multirow{3}{*}{ Cache.db } & $\begin{array}{l}\text { This database store user name } \\
\text { information (username) a local } \\
\text { directory path that stores information } \\
\text { about files stored in CloudMe, } \\
\text { information about the names, size, } \\
\text { and other metadata. }\end{array}$ \\
\hline YYYY-MM- & $\begin{array}{l}\text { This file log Shows the login time } \\
\text { information to connect to the } \\
\text { Internet, connections problem, and } \\
\text { DD.txt }\end{array}$ \\
\hline
\end{tabular}

\subsubsection{Copy}

Copy client during the installation and running of applications using the Diskpulse and Process monitor easily find where the main file Copy stored during the analysis found important files related or could be evidence.

Fig 5 is shown the location on the path to the directory where the log files and database copy.

\begin{tabular}{|c|c|}
\hline Т8g & Flle \\
\hline copy & limg_cloudtest1.dd/Users/cloudtes2015/AppData/Roaming/Copy/gynclog.tht \\
\hline copy & limg_doudtest1.dd/Users/cloudtest2015/AppData/Roaming/Copy/trgce.bt \\
\hline copy & limg_cloudtest1.dd/Users/cloudtest2015/AppData/Roaming/Copy/copy mir888659@gmail.com.db \\
\hline copy & limg_cloudtest1.dd/Users/cloudtest2015/AppData/Roaming/Copy/config.db \\
\hline
\end{tabular}

Fig 5: Location File of Copy
In Table 7 is shown the name of the database and log files on Copy and its description.

Table 7. File Analysis of Copy

\begin{tabular}{|l|l|}
\hline \multicolumn{1}{|c|}{ Name File } & \multicolumn{1}{|c|}{ Description } \\
\hline \multirow{2}{*}{ config.db: } & $\begin{array}{l}\text { File database saving settings such as } \\
\text { the user email, first name, last name, } \\
\text { and user ID, ClientID. It also contains } \\
\text { the path root and the cache root } \\
\text { directory of Copy's, client version } \\
\text { and other settings. }\end{array}$ \\
\hline \multirow{5}{*}{ trace.txt: } & $\begin{array}{l}\text { File log file's entries contain } \\
\text { information related the hosting } \\
\text { machine (operating system, host } \\
\text { information, etc.), the client } \\
\text { application, and the server. }\end{array}$ \\
\hline synclog.txt & $\begin{array}{l}\text { This files log store information } \\
\text { related to the operations types. Some } \\
\text { operations are authentication attempts } \\
\text { and file manipulation (such as upload, } \\
\text { download, modification, and } \\
\text { deletion). }\end{array}$ \\
\hline copy & $\begin{array}{l}\text { This file database is very interesting } \\
\text { for a investigator this point it } \\
\text { contains the list of files and metadata } \\
\text { stored in the root directory of Copy. } \\
\text { (in our case the name of the file is } \\
\text { mir968659@gmail.com.db) }\end{array}$ \\
\hline User_Email $>$ db
\end{tabular}

\subsubsection{Dropbox}

Dropbox application to open the database file must use tools dropboxdescrytor to be able to read this tool database file can be downloaded at magnetforensics.com

Fig 6 is shown the location on the path to the directory where the $\log$ files and database Dropbox.

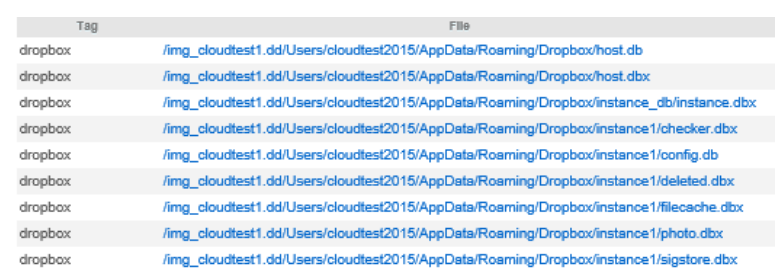

Fig 6: Location File of Dropbox

In Table 8 is shown the names of the database on Dropbox and its Description.

Table 8. File Analysis of Dropbox

\begin{tabular}{|l|l|}
\hline Name File & \multicolumn{1}{|c|}{ Description } \\
\hline config.dbx & $\begin{array}{l}\text { This files database at the table config } \\
\text { stores the host IDs, the user's email, } \\
\text { user information, host_id, root folder } \\
\text { paths, among other settings. This file } \\
\text { is one of the encrypted files, and } \\
\text { after it has been decrypted with } \\
\text { magnet forensics dropbox decryptor } \\
\text { it is possible to see it. }\end{array}$ \\
\hline filecache.dbx & $\begin{array}{l}\text { This file database contains the server } \\
\text { paths, the files lists and the files } \\
\text { names, the sizes of the files, the } \\
\text { modification and creation times in } \\
\text { the table file_journal. The table } \\
\text { deleted_fileids contain metadata file } \\
\text { has been deleted. }\end{array}$ \\
\hline
\end{tabular}




\subsection{Memory Analysis}

Memory analysis using Belkasoft Evidence Finder and a search keywords used in the file .mem. in memory there is a lot of information that can be used and proof. However memory analysis is not always possible, and therefore the possibility of memory can not be analyzed during digital forensic investigations. If possible, the memory should be acquired. Digital evidence can be found in memory, among others include, user information, user details, a list of files and information files, accessing files and folders, process information, procces instruction, host names, libraries are loaded, libraries are loaded via the server, temporary files, accessing database files and log files, authentication, artifact using web browser also found in ram such as cookies, URL history and other in Fig. 7 shows the result of analysis using WinHex.

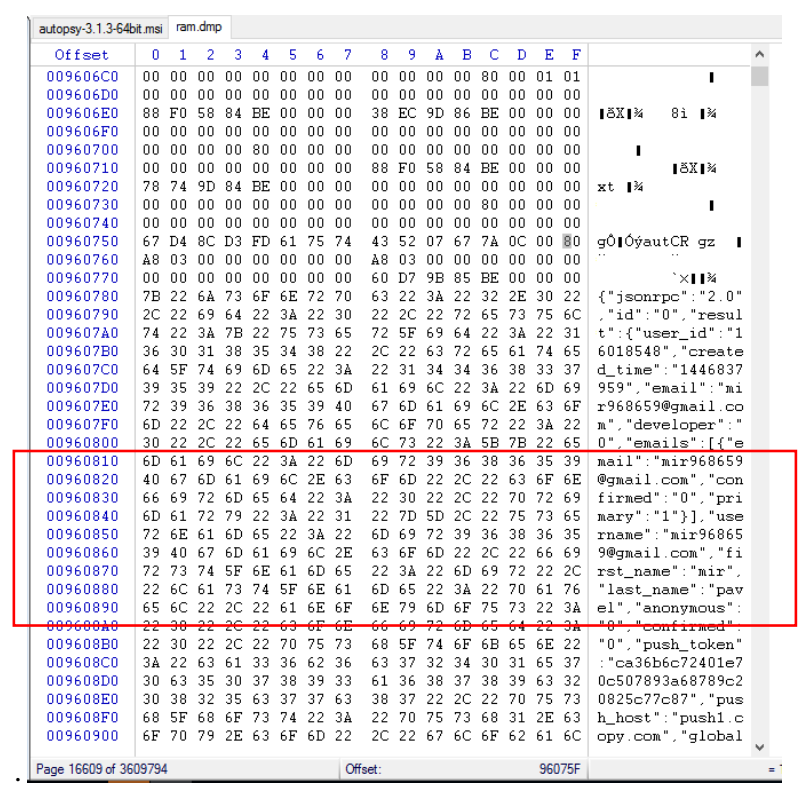

\section{Fig 7: Analysis RAM using WinHex}

In the picture above is based on analyzes using WinHex looks username, email, user information and other.

\subsection{Deleted Analysis \& Uninstall Analysis}

One thing that is important to conduct digital investigations, is recovering from a deleted file. For the recovering deleted files from cloud storage accounts, perform recovery from the server side and the client side. Copy the client application has a feature known as undelete. This feature can restore a deleted file either locally or on a server.

CloudMe and Dropbox does not have a similar feature, but it can be done, restore deleted files or files that are modified, which account can only be accessed via a web browser is not through the application as well as on Copy. And differences keep deleted files in days. Dropbox can also delete files permanently.

Using tools Autopsy, File (allocated space) is still visible in recyble bin, and point to the directory of origin, before dideleted. The files can still be recovered. And if the file has been deleted in recyble bin (unallocated space) still can be recovered, using tools Foremost, SCAPEL, and PhotoRec TSK toolkit.

The uninstall process on CloudMe, the main folder to store database and $\log$ files in the folder C:luserlcloudtest2015 AppDatalLocallCloudMelSync still visible as well as files synchronization is still contained in the folder C:luser/ cloudtest2015\CloudMe, use Scanreg can find registry files related to the use of applications CloudMe.

After doing the uninstall process, the main folder of the Copy also includes files that are inside the folder C:luser/ cloudtest2015\AppDatalRoaminglCopy also still there, while the database and log files have been deleted. This means that we can not see the user usage activity. using RegScanner we can use string-based search using the copy, it is possible to find some registry keys were left behind when the application has been deleted and uninstalled.

During the uninstall process of the dropbox, dropbox folder is still visible on the hard disk, it also still contains the files. the folder C:IUserslcloudtest2015\AppData\Roaming\Dropbox is also still there, while the files are encrypted database has been deleted. There are many registry key from dropbox and can be traced remaining data during the installation process, it can be used as evidence of the use of client applications. 


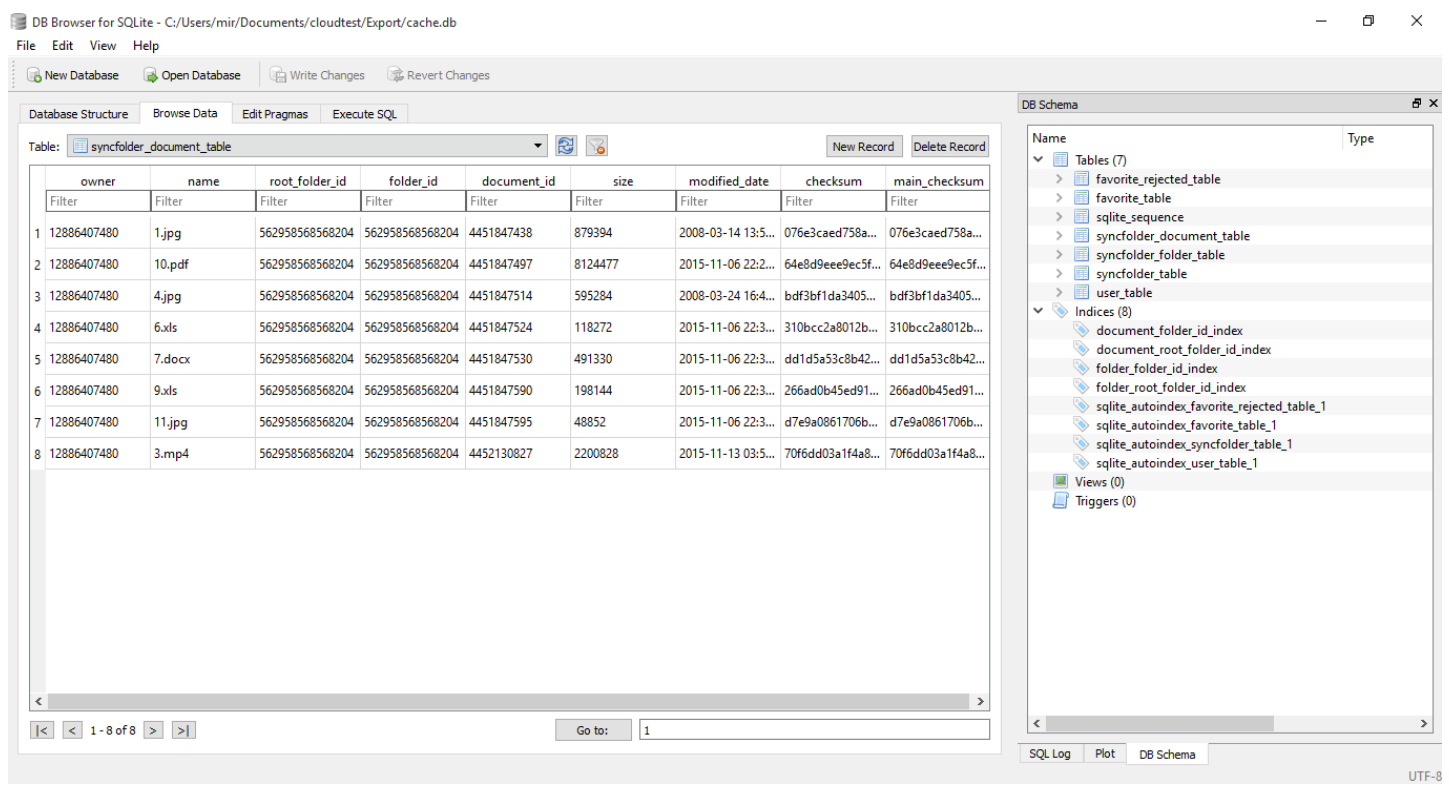

Fig 8: Table syncfolder_document_file in database Cache.db of CloudMe Application

In Fig. 8 is the content of a database file from an application cache.db CloudMe, shows the remaining files, and files that have been deleted does not appear in the table syncfolder_document_tabel that file 2.jpg, and file $8 . \mathrm{mp} 3$ 5.jpg file.

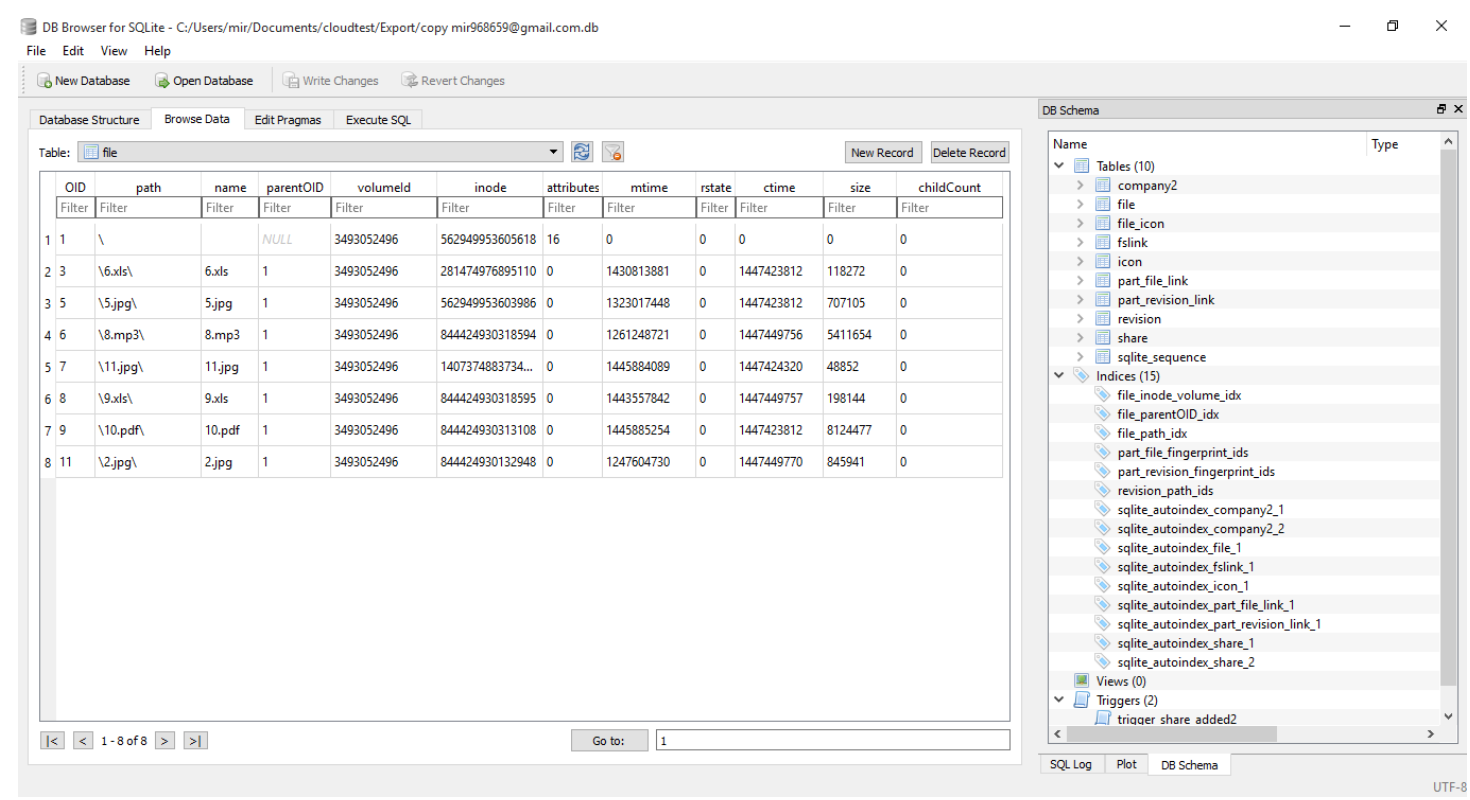

Fig 9: Table file in database mir968659@gmail.com.db of Copy Application

In Fig.9 is a database file contents of the database mir968659 @ gmail.com.db of application Copy, shows the remaining files, and files that have been deleted does not appear in the table file namely 1.jpg file, file 4.jpg and file 7.docx. 


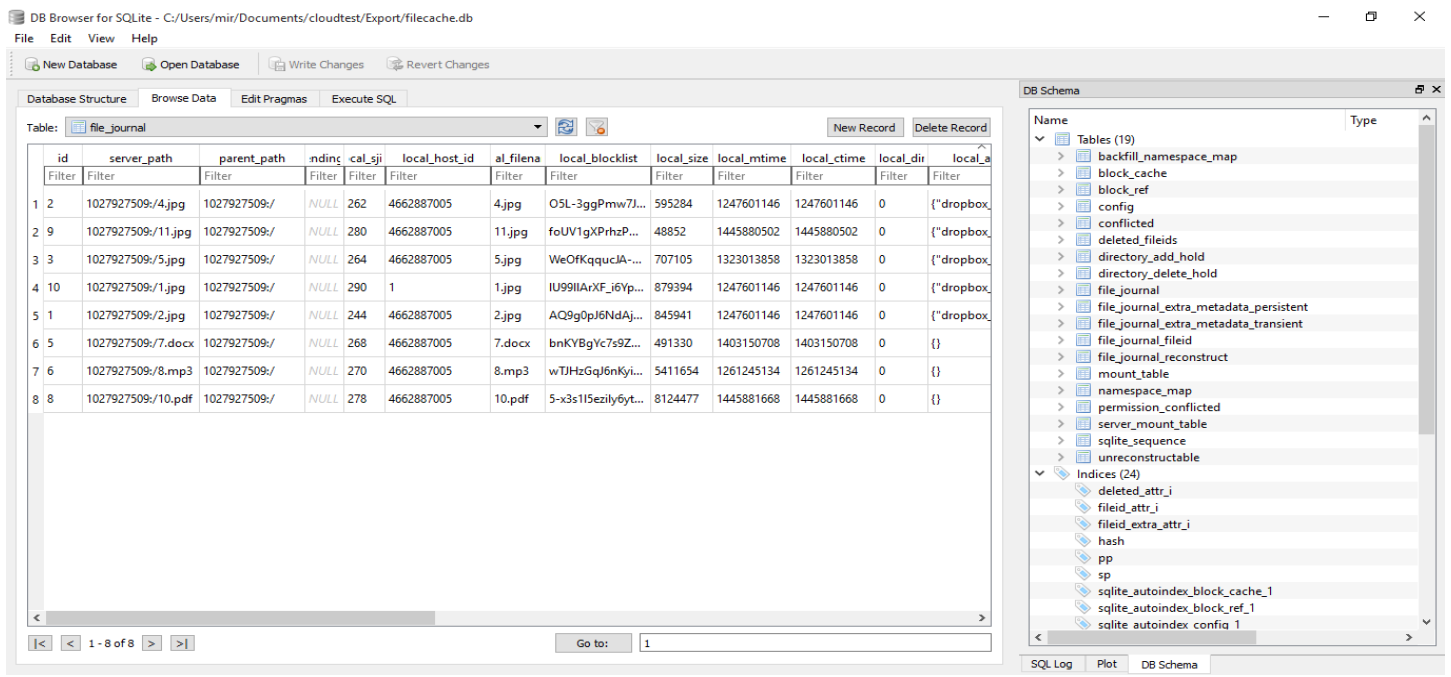

Fig 10: Table file_journal in filecache.db of Dropbox Application

In Fig.10 is a file from the contents of the application Dropbox filecache.db database, shows the remaining files, and files that have been deleted does not appear in the table file_journal namely 3.mp4 file, file 6.xls and file 9.xls

\section{CONCLUCION}

In this paper presented that it is possible of getting digital evidence that related to the use of cloud storage client on Windows 10 platform. Digital evidence can be found in the database and $\log$ files created by applications, on the web browser, in memory and the registry. This study contributes to the problems of the cloud in particular helps forensic investigators in mapping the area that became a source of digital evidence. Based on the test results success rate average is $82,63 \%$ and the remaining can not be analyzed, the results depend on various state, procedures and tools that are used, the research can be carried out smoothly.

\section{REFERENCES}

[1] Cisco.com, "No Title," 2015. [Online]. Available: a) http://newsroom.cisco.com/press-releasecontent?type $=$ webcontent $\&$ articleId $=1724918$. [Accessed: 01-Nov-2015].

[2] Cisco, "Cisco Global Cloud Index: Forecast and Methodology, 2014-2019," Cisco Press, 2015. [Online]. Available:

http://www.cisco.com/c/en/us/solutions/collateral/service -provider/global-cloud-indexgci/Cloud_Index_White_Paper.pdf. [Accessed: 01-Nov2015].

[3] D. Quick, B. Martini, and K.-K. R. Choo, Cloud Storage Forensics. 2014

[4] M. Epifani, "Cloud Storage Forensics," in SANS Eurupean Digital Forensics Summit 2013, 2013.

[5] J. Chung, H., Park and C. Lee, S., Kang, "Digital Forensic Investigation of Cloud Storage Services, Digital Investigation," 2012.

[6] S. Mehreen and B. Aslam, "Windows 8 cloud storage analysis: Dropbox forensics," in 2015 12th International Bhurban Conference on Applied Sciences and Technology (IBCAST), 2015, pp. 312-317.
[7] R. Malik, "Analysis of Evidence in Cloud Storage Client Applications on the Windows Platform," Int'l Conf. Secur. Manag., 2015.

[8] M. Borgmann, T. Hahn, M. Herfert, T. Kunz, M. Richter, U. Viebeg, and S. Vowé, On the Security of Cloud Storage Services. Fraunhofer Verlag; Stuttgart, Germany: Fraunhofer Institute for Secure Information Technology SIT, 2012.

[9] T. Prasath and S. Karthikeyan, "Cloud Storage Vendors Wide Support and Security Key Features for Shifting Towards Business Perspective," Int. J. Cloud Comput. Serv. Sci., vol. 2, no. 6, pp. 421-426, 2013.

[10] M. Lohnash and G. Akerlund, "Online Backup Comparison," $2015 . \quad$ [Online]. Available: http://www.backupreview.com/online-comp-provider/. [Accessed: 01-Nov-2015]

[11] G. Reese, "Cloud Forensics Using Ebs Boot Volumes," Oreilly.com, 2010.

[12] A. Iswardani and I. Riadi, "Denial of service log analysis using density k-means method," J. Theor. Appl. Inf. Technol., vol. 83, no. 2, p. 2, 2016.

[13] P. . Clark, "Digital Forensics Tool Testing-Image Metadata in the Cloud', Department of Computer Science and Media Technology," Gjøvik Univ. Coll., 2011.

[14] A. K. Mccurdy, "Dropbox: Forensic Detection the requirements of Edinburgh Napier University," 2013.

[15] H. Chung, J. Park, S. Lee, and C. Kang, "Digital forensic investigation of cloud storage services," Digit. Investig., vol. 9, no. 2, pp. 81-95, 2012.

[16] Www.dropbox.com, "No Title." [Online]. Available: http://www.dropbox.com.

[17] Copy.com, "No Title." [Online]. Available: http://www.copy.com.

[18] Www.cloudme.com, "No Title." [Online]. Available: http://www.cloudme.com. 